\title{
APLICAÇÃO DA ANÁLISE HARMÔNICA POR SÉRIES DE FOURIER PARA A PREVISÃO DE PRODUTIVIDADE DA CULTURA DO CAFÉ NO ESTADO DE MINAS GERAIS ${ }^{1}$
}

\author{
LUIZ G. DE CARVALHO ${ }^{2}$, GILBERTO C. SEDIYAMA ${ }^{3}$, PAULO R. CECON ${ }^{4}$, \\ HELENA M. R. ALVES ${ }^{5}$
}

RESUMO: O objetivo deste trabalho foi obter um modelo de previsão de produtividade para a cultura do café, em sete municípios do Estado de Minas Gerais. Submeteram-se à análise harmônica por séries de Fourier, séries de produtividades representativas de cada município, das quais se extraíram os coeficientes até o sétimo harmônico, submetendo-os à regressão linear múltipla nos três primeiros componentes principais de um conjunto de 33 variáveis inerentes à produção cafeeira. Essas variáveis foram médias de 15 anos correspondentes aos mesmos anos das produtividades e subdivididos em quatro períodos trimestrais, ao longo do ciclo produtivo da cultura (julho a junho). O modelo mostrouse inconsistente, apresentando erros das estimativas bastante discrepantes, evidenciando a complexidade de modelagem de previsão de safras para a cultura do café.

PALAVRAS-CHAVE: modelagem agrometeorológica, componentes principais, balanço hídrico.

\section{FORECAST OF COFFEE CROP PRODUCTIVITY BY HARMONIC ANALYSIS, USING THE FOURIER SERIES IN MINAS GERAIS STATE, BRAZIL}

\begin{abstract}
The objective of this work was to obtain a forecast model of coffee crop productivity in seven municipal districts of Minas Gerais State, Brazil. The harmonic analysis in Fourier series was applied on productivity series to each location. The obtained coefficients until the harmonic seventh were submitted to multiple linear regression in the first three principal components of a group of 33 inherent variables of coffee production. These variables were averages of 15 years corresponding to the same years of productivity and subdivided in four quarterly periods during the agricultural year (July to June). The model did not prove adequate, since the results presented errors of estimatives with great discrepancies evidencing the complexity of yield forecast of coffee crop.
\end{abstract}

KEYWORDS: agrometeorological modeling, principal components, water balance.

\section{INTRODUÇÃO}

Pela sua grande importância econômica, a previsão de produtividade é de grande valia, pois constitui informação extremamente útil para permitir ao agricultor e ao País melhor planejamento nas atividades do agronegócio. Contudo, a previsão de safras agrícolas no Brasil tem sido um grande problema, particularmente para a cultura do café. O extinto Instituto Brasileiro do Café (IBC) desempenhava a contento esse tipo de serviço até 1990. Atualmente, a responsabilidade oficial de previsão de safra cafeeira está a cargo da Companhia Nacional de Abastecimento (CONAB), vinculada ao Ministério da Agricultura, Pecuária e Abastecimento (MAPA).

A produtividade da cultura do café sofre oscilações devido, principalmente, aos fatores climáticos e pelo efeito da bienalidade de produção, que é uma característica fisiológica própria dessa

\footnotetext{
1 Extraído da tese de doutorado do primeiro autor. Projeto financiado pelo CNPq, Proc. N. 474244/2001-6 (NV), mod. APQ.

2 D.S., Prof. Adjunto, DEG, Universidade Federal de Lavras, Lavras - MG, lgonsaga@ ufla.br

${ }^{3}$ Ph.D., Professor Titular, DEA/UFV, sediyama@ufv.br

4 D.S., Professor Titular, DPI/UFV, cecon@dpi.ufv.br

5 Ph.D., Pesquisadora, EMBRAPA, helena@ufla.br

Recebido pelo Conselho Editorial em: 4-6-2003

Aprovado pelo Conselho Editorial em: 24-10-2005
} 
cultura (RENA \& MAESTRI, 1985), ampliando a complexidade da previsão. HOOGENBOOM (2000) apresentou ampla discussão sobre a contribuição da agrometeorologia em modelos de simulação do crescimento, desenvolvimento e produção das culturas, sendo os elementos climáticos a chave de entrada requerida para a operação da maioria desses modelos. WEILL (1990) verificou que os elementos climáticos são os que individualmente explicam a maior parte da variabilidade total da produção do cafeeiro do Estado de São Paulo, em comparação com fatores edáficos e biológicos.

Quanto à disponibilidade de água no solo, deficiência hídrica severa mostra-se crítica para o cafeeiro nos estádios vegetativo, de formação dos grãos e de maturação, nas condições do Estado de São Paulo (CAMARGO, 1987). No período de dormência das gemas, a deficiência hídrica moderada é benéfica, permitindo florada intensa e uniforme após a chuva ou irrigação. De acordo com RENA \& MAESTRI (1985), a floração propriamente dita é provocada pelas primeiras chuvas da estação após o período seco. Depois da florada, deficiências e excessos de água predispõem os cafeeiros à atrofia.

PICINI (1998) parametrizou e testou modelos agrometeorológicos que relacionam a fenologia, a bienalidade e a produtividade do cafeeiro Mundo Novo, nas condições do Estado de São Paulo. Os modelos basearam-se na penalização da produtividade potencial em função da produtividade do ano anterior e das relações ETR/ETP (evapotranspirações real e potencial). O modelo com melhores resultados, após algumas modificações, foi o aditivo, desenvolvido por STEWART et al. (1976).

Para o estudo de séries temporais que apresentam variações periódicas sazonais, pode-se recorrer à análise harmônica por séries de Fourier para o desenvolvimento de modelos. Como a cultura do café apresenta o efeito da bienalidade, inerente a sua fisiologia, pode ser considerada como uma cultura de produção periódica, sendo possível aplicar a análise harmônica para a previsão de produtividade.

Outra técnica bastante útil em modelagem é a análise estatística multivariada de componentes principais. Esses são independentes entre si e estimados com o propósito de reter, em ordem decrescente, o máximo de informação, em termos de variação total contida nos dados iniciais, permitindo reduzir a dimensão do conjunto original (CRUZ \& REGAZZI, 1997). Dessa forma, podese, na maioria das vezes, e principalmente quando se tem o número de observações inferior à quantidade de variáveis, fazer a regressão linear com os componentes principais que retiveram a maior parte da variância original dos dados, desde que sejam suficientes para representar as variáveis originais, reduzindo substancialmente as variáveis regressoras dentro do modelo linear de regressão múltipla. Contudo, cada componente principal é uma função linear de todas as variáveis originais, tendo-se, ainda, essas variáveis como entrada do modelo. Descrições detalhadas da regressão em componentes principais podem ser encontradas, entre outros autores, em HAAN (1977).

Por fim, nota-se que o desenvolvimento e/ou a adaptação de uma metodologia alternativa para a previsão de safras, com o objetivo de reduzir os custos e, conseqüentemente, obter maior agilidade no levantamento de dados, vem sendo bastante pertinente para os tempos atuais.

Diante do exposto, este trabalho teve como objetivo avaliar a aplicação da análise harmônica por séries de Fourier de senos e cossenos, para a previsão de produtividades da cultura do café no Estado de Minas Gerais.

\section{MATERIAL E MÉTODOS}

Os dados de produtividade da cultura do café (Tabela 1) foram obtidos no Instituto Brasileiro de Geografia e Estatística (IBGE), os quais são registrados no "Produção Agrícola Municipal - Culturas Temporárias e Permanentes" (PAM). 
TABELA 1. Coordenadas geográficas e séries anuais de produtividades de café em coco $\left(\mathrm{kg} \mathrm{ha}^{-1}\right)$ de municípios de Minas Gerais utilizadas na avaliação do modelo pela análise harmônica por séries de Fourier.

\begin{tabular}{|c|c|c|c|c|c|c|c|}
\hline \multirow{2}{*}{$\begin{array}{c}\text { Coord. } \\
\text { Geográficas }\end{array}$} & \multicolumn{7}{|c|}{ Municípios } \\
\hline & Alfenas & Guaxupé & Lavras & Monte Belo & S. S. Paraíso & Varginha & Viçosa \\
\hline Latitude & $21^{\circ} 18^{\prime}$ & $21^{\circ} 17^{\prime}$ & $21^{\circ} 14^{\prime}$ & $21^{\circ} 21^{\prime}$ & $20^{\circ} 54^{\prime}$ & $21^{\circ} 31^{\prime}$ & $20^{\circ} 45^{\prime}$ \\
\hline Longitude & $45^{\circ} 56^{\prime}$ & $46^{\circ} 40^{\prime}$ & $45^{\circ} 00^{\prime}$ & $46^{\circ} 14^{\prime}$ & $47^{\circ} 04^{\prime}$ & $45^{\circ} 30^{\prime}$ & $42^{\circ} 51^{\prime}$ \\
\hline Altitude (m) & 818,83 & 920,00 & 918,84 & 826,00 & 967,33 & 900,00 & 689,73 \\
\hline \multicolumn{8}{|l|}{ Ano Agrícola } \\
\hline $1985 / 1986$ & 1.372 & 293 & 683 & 614 & 1.472 & 643 & 1.015 \\
\hline $1986 / 1987$ & 2.880 & 1.073 & 2.300 & 1.127 & 2.075 & 2.436 & 1.140 \\
\hline 1987/1988 & 1.739 & 1.120 & 1.193 & 900 & 1.266 & 720 & 2.200 \\
\hline $1988 / 1989$ & 2.400 & 1.240 & 1.208 & 1.130 & 945 & 1.800 & 1.440 \\
\hline 1989/1990 & 1.202 & 1.022 & 1.287 & 567 & 947 & 1.244 & 833 \\
\hline 1990/1991 & 1.800 & 1.159 & 1.300 & 1.800 & 948 & 2.160 & 1.500 \\
\hline $1991 / 1992$ & 1.344 & 1.238 & 1.300 & 1.200 & 1.100 & 1.440 & 1.500 \\
\hline $1992 / 1993$ & 3.000 & 1.238 & 1.300 & 1.511 & 1.280 & 1.800 & 1.500 \\
\hline 1993/1994 & 2.899 & 1.146 & 1.300 & 1.763 & 1.558 & 1.200 & 1.500 \\
\hline $1994 / 1995$ & 1.558 & 790 & 959 & 1.456 & 616 & 638 & 1.529 \\
\hline $1995 / 1996$ & 3.335 & 1.142 & 1.680 & 2.142 & 2.563 & 600 & 930 \\
\hline 1996/1997 & 1.800 & 2.160 & 1.440 & 1.500 & 1.244 & 600 & 1.500 \\
\hline 1997/1998 & 3.600 & 2.160 & 2.040 & 2.400 & 2.400 & 600 & 1.500 \\
\hline $1998 / 1999$ & 1.824 & 1.188 & 1.800 & 2.074 & 1.200 & 1.440 & 1.500 \\
\hline $1999 / 2000$ & 2.400 & 1.188 & 2.060 & 2.160 & 1.440 & 1.440 & 1.500 \\
\hline Média & 2.210 & 1.210 & 1.457 & 1.490 & 1.404 & 1.251 & 1.406 \\
\hline
\end{tabular}

Cada série de dados da produtividade cafeeira da Tabela 1 foi submetida à análise harmônica por séries de Fourier de senos e cossenos, conforme a expressão seguinte, cuja descrição detalhada dessa metodologia é apresentada por ASSIS et al. (1996).

$$
\hat{\mathrm{Y}}_{\text {est }}=\mathrm{Y}_{0}+\sum_{\mathrm{n}=1}^{7}\left(\mathrm{a}_{\mathrm{n}} \cos n \omega \mathrm{t}_{\mathrm{i}}+\mathrm{b}_{\mathrm{n}} \operatorname{sen} n \omega \mathrm{t}_{\mathrm{i}}\right)
$$

em que,

$\hat{\mathrm{Y}}_{\text {est }}$ - produtividade estimada;

$\mathrm{Y}_{0}$ - coeficiente das séries de Fourier conhecido por harmônico fundamental, representando a própria média aritmética da série de dados observados;

$\mathrm{n}-1,2, \ldots, 7$ identifica o número de harmônicos que, para as séries analisadas, varia de 1 a 7 ;

$a_{n}$ e $b_{n}$ - respectivos coeficientes de cosseno e seno para o harmônico "n" das séries de Fourier;

$\omega$ - freqüência angular da onda de produtividade, sendo $\omega=2 \pi / \mathrm{T}$ e $\mathrm{T}$ o período, em que este é correspondente ao número de dados observados, ou seja, neste trabalho $\mathrm{T}=15$, e

$\mathrm{t}_{\mathrm{i}}$ - ordenação numérica dos anos de produtividades correspondentes da série $(\mathrm{i}=0,1, \ldots, \mathrm{T}-1)$.

Os coeficientes $Y_{0}, a_{n}$ e $b_{n}$ foram obtidos, respectivamente, pelas seguintes expressões:

$$
\mathrm{Y}_{0}=\frac{1}{\mathrm{M}} \sum_{\mathrm{i}=1}^{\mathrm{M}} \mathrm{Y}_{\mathrm{i}} \quad \text { (2) } \quad \mathrm{a}_{\mathrm{n}}=\frac{2}{\mathrm{M}} \sum_{\mathrm{i}=1}^{\mathrm{M}} \mathrm{Y}_{\mathrm{i}} \cos \mathrm{n \omega t} \mathrm{t}_{\mathrm{i}}
$$

$$
\mathrm{b}_{\mathrm{n}}=\frac{2}{\mathrm{M}} \sum_{\mathrm{i}=1}^{\mathrm{M}} \mathrm{Y}_{\mathrm{i}} \operatorname{sen} \mathrm{n \omega t} \mathrm{t}_{\mathrm{i}}
$$

sendo $\mathrm{M}=15$, ou seja, o número de dados na série, e $\mathrm{Y}_{\mathrm{i}}$ a produtividade observada do i-ésimo ano.

A eq.(1) pode, também, ser expressa somente em termos de cosseno, como se segue: 


$$
\hat{\mathrm{Y}}_{\mathrm{est}}=\mathrm{Y}_{0}+\sum_{\mathrm{n}=1}^{7} \mathrm{~A}_{\mathrm{n}} \cos \left(\mathrm{n \omega t}_{\mathrm{i}}-\phi_{\mathrm{n}}\right)
$$

sendo,

$$
A_{n}=\sqrt{a_{n}^{2}+b_{n}^{2}}
$$

$$
\phi_{\mathrm{n}}=\tan ^{-1}\left(\frac{\mathrm{b}_{\mathrm{n}}}{\mathrm{a}_{\mathrm{n}}}\right)
$$

em que, $A_{n}$ e $\phi_{n}$ são, respectivamente, a amplitude e o ângulo de defasagem do n-ésimo harmônico. $\mathrm{O}$ quadrante ao qual pertence $\phi_{n}$ é determinado pelos sinais dos coeficientes $a_{n}$ e $b_{n}$ (ASSIS et al., 1996).

O número de harmônicos ajustados pelas séries de Fourier para cada conjunto de dados foi igual a 7, ou seja, (n-1)/2, pois as séries contêm um conjunto ímpar equivalente a 15 observações.

Além das outras variáveis (descritas adiante) utilizadas para a avaliação do modelo, acrescentouse a produtividade média (PM) de cada série de dados por município, a qual foi submetida à análise de Fourier. Uma vez obtido o modelo, a PM passou a ser a produtividade do ano anterior (Ya) como variável de entrada, ao prever a produtividade do ano seguinte.

Para cada município, efetuou-se o cálculo do balanço hídrico no solo (seriado mensal) para os 15 anos, de acordo com os dados de produtividade e conforme metodologia proposta por THORNTHWAITE \& MATHER (1955), apresentada por PEREIRA et al. (1997). A capacidade de água disponível no solo (CAD) foi de $100 \mathrm{~mm}$, também utilizada por MATIELLO (1991) e PICINI (1998). O processamento desses dados, assim como dos componentes do balanço hídrico, foi separado conforme as fases fenológicas do cafeeiro, sendo assim definidas (MATIELLO, 1991): Fase 1 - julho a setembro; Fase 2 - outubro a dezembro; Fase 3 - janeiro a março, e Fase 4 - abril a junho.

Os dados utilizados, concomitantes ao mesmo período das séries anuais de produtividades, foram representados pelas respectivas médias de cada fase fenológica. Tais dados, dessa forma definidos, foram: temperaturas máximas $\left(\mathrm{Tx}_{1}, \mathrm{Tx}_{2}, \mathrm{Tx}_{3}, \mathrm{Tx}_{4}\right)$, temperaturas mínimas $\left(\mathrm{Tn}_{1}, \mathrm{Tn}_{2}, \mathrm{Tn}_{3}, \mathrm{Tn}_{4}\right)$, amplitudes de temperaturas $\left(\mathrm{A}_{1}, \mathrm{~A}_{2}, \mathrm{~A}_{3}, \mathrm{~A}_{4}\right)$, temperaturas médias $\left(\mathrm{Tm}_{1}, \mathrm{Tm}_{2}, \mathrm{Tm}_{3}, \mathrm{Tm}_{4}\right)$, precipitação pluvial $\left(\mathrm{P}_{1}, \mathrm{P}_{2}, \mathrm{P}_{3}, \mathrm{P}_{4}\right)$, evapotranspiração real $\left(\mathrm{ETR}_{1}, \mathrm{ETR}_{2}, \mathrm{ETR}_{3}, \mathrm{ETR}_{4}\right)$ e deficiência hídrica $\left(\mathrm{DH}_{1}\right.$, $\mathrm{DH}_{2}, \mathrm{DH}_{3}$ e $\mathrm{DH}_{4}$ ). Foram, ainda, calculadas as médias por períodos de exposições diárias à radiação solar durante o ano para os municípios que compõem o modelo, conforme ALVES et al. (1983), originando as variáveis $\mathrm{ERS}_{1}, \mathrm{ERS}_{2}, \mathrm{ERS}_{3}$ e ERS 4 . Os índices 1; 2; 3 e 4 representam as fases fenológicas anteriormente descritas. Assim, por exemplo, para a primeira variável $\mathrm{Tx}_{1}$, inicialmente calculou-se a média trimestral da temperatura máxima de julho a setembro de cada ano, durante os 15; da média desses 15 valores, gerou-se a variável $\mathrm{Tx}_{1}$. Esse procedimento, à exceção de Ya, é válido para todas as demais variáveis. Portanto, o conjunto das variáveis ficou definido por uma matriz de 33 x 7, ou seja, 33 variáveis, tendo cada uma sete observações (sete municípios). De posse do modelo, as variáveis utilizadas para sua avaliação passaram a ser as de entrada.

Como foram submetidas à análise de Fourier sete séries de dados de produtividade, obtiveramse, portanto, para cada coeficiente de ordem " $n$ ", seja $a_{n}$ ou $b_{n}$, sete valores correspondentes. Considerando que, para cada um desses coeficientes com sete repetições, existe um conjunto de 33 variáveis, torna-se sem possibilidade de, estatisticamente, efetuar a análise de regressão linear múltipla de cada coeficiente, em função dessas variáveis, pois o número de parâmetros a serem estimados é superior ao de observações. Portanto, adotou-se a técnica de análise multivariada de componentes principais para transformar essas variáveis em um novo conjunto dessas, ou seja, os chamados escores dos componentes principais, permitindo, assim, a execução das regressões. Além disso, essa técnica permite selecionar variáveis, adotando-se o critério de eliminar aquelas que possuem maiores correlações com os componentes principais de menor importância. Como essas apresentam magnitudes diferenciadas, conforme as grandezas a que se referem, a análise de componentes principais foi efetuada com base na matriz de correlação dessas. 
Os coeficientes obtidos pelo ajuste das séries de Fourier aos dados de produtividade $\left(\mathrm{Y}_{0}, \mathrm{a}_{\mathrm{n}}\right.$ e $\left.\mathrm{b}_{\mathrm{n}}\right)$, foram separados e submetidos, como variáveis dependentes, à análise de regressão linear múltipla, em função dos escores dos componentes principais retidos. Voltando-se à eq.(1), os coeficientes $\mathrm{Y}_{0}, \mathrm{a}_{\mathrm{n}} \mathrm{e}$ $\mathrm{b}_{\mathrm{n}}$ foram substituídos pelas respectivas regressões lineares múltiplas, gerando o modelo prognóstico de produtividade de café para a região representada pelos sete municípios analisados.

O teste foi realizado, utilizando dados de produtividade independentes (de outro município), os quais não foram empregados para avaliar o modelo, conforme cita PICINI (1998). Tais dados foram obtidos do município de Três Pontas, próximo ao de Varginha. Por indisponibilidade de dados climáticos para aquele município, adotaram-se os adquiridos para Varginha, podendo considerar ambos os municípios com características climáticas semelhantes, conforme observado no trabalho de ASPIAZÚ et al. (1990). Os dados de determinado ano agrícola foram utilizados para estimar a produtividade do ano seguinte, sendo, portanto, o dado de produtividade desse o valor esperado.

Como a análise de componentes principais foi realizada com base na matriz de correlação das 33 variáveis, essas tornam-se padronizadas. Portanto, para o ano da previsão, os dados de entrada do modelo devem também ser padronizados, conforme a equação seguinte:

$$
X_{\text {ip }}=\frac{X_{i}-\bar{X}}{s(X)}
$$

em que,

$\mathrm{X}_{\mathrm{ip}}$ - i-ésima variável padronizada para o ano de previsão;

$\mathrm{X}_{\mathrm{i}}$ - i-ésima variável para o ano de previsão, e

$\overline{\mathrm{X}}$ e $\mathrm{s}(\mathrm{X})$ - média e desvio-padrão, respectivamente, das observações de cada variável $\mathrm{X}_{\mathrm{i}}$ que originou o modelo.

Os valores de produtividade estimados e observados foram comparados, aplicando-se o erro relativo percentual $(\mathrm{ERP})$ :

$$
\mathrm{ERP}=100 \frac{\text { Yest }- \text { Yobs }}{\text { Yobs }}
$$

em que,

Yest - produtividade estimada, sacas ha ${ }^{-1}$, e

Yobs - produtividade observada, sacas ha ${ }^{-1}$.

Calcularam-se, também, as relações entre produtividades estimadas e observadas com a potencial (Yest/Yp e Yobs/Yp), cujos valores foram plotados graficamente em escala de 0 a 1.

A produtividade potencial (Yp) indica o potencial produtivo da cultura, desde que não ocorra nenhum fator restritivo. KANEMASU (1983) propôs que, de uma série de dados de produtividade, a maior delas fosse acrescida de $10 \%$, com o objetivo de eliminar todo o efeito do ambiente. Adotandose esse critério, da série para teste do modelo (dados de Três Pontas - MG), a maior produtividade foi de $2.054 \mathrm{~kg} \mathrm{ha}^{-1}$, sendo, portanto, a produtividade potencial equivalente a $2.259 \mathrm{~kg} \mathrm{ha}^{-1}$.

Procedeu-se à análise de regressão simples (Yest/Yp x Yobs/Yp), com a reta passando pela

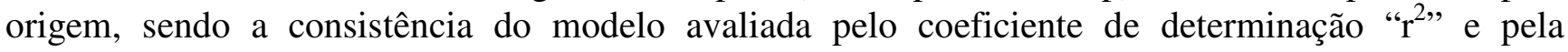
concordância expressa pelo índice "d" proposto por WILLMOTT et al. (1985), conforme eq.(10). O índice "d", com variação entre 0 e 1, indica o grau de exatidão entre valores estimados e observados, com a ressalva de que, quanto mais próximo de um (1), melhor a exatidão do modelo em prever a variável dependente, ao passo que o " $\mathrm{r}^{2}$ " indica a precisão do modelo, ou seja, quanto da variação da variável dependente é explicada por aquela das variáveis independentes. 


$$
d=1-\left[\frac{\sum_{i=1}^{n}\left(\text { Yest }_{i}-\text { Yobs }_{i}\right)^{2}}{\sum_{i=1}^{n}\left(\mid \text { Yest }_{i}-\bar{Y}_{\text {obs }}|+| \text { Yobs }_{i}-\bar{Y}_{\text {obs }} \mid\right)^{2}}\right]
$$

em que,

Yest $_{i}$ - i-ésimo valor previsto ou estimado;

Yobs $_{\mathrm{i}}$ - i-ésimo valor observado, e

Yobs - média dos valores observados.

\section{RESULTADOS E DISCUSSÃO}

A tentativa de eliminar algumas das variáveis do conjunto original dessas, pelo critério de redução daquelas que possuíam maiores correlações com os componentes principais de menor importância, não foi eficiente, pois as insignificâncias entre elas não foram bem definidas. Sendo assim, manteve-se o mesmo conjunto original composto pelas 33 variáveis para a entrada do modelo. Os três primeiros componentes principais do conjunto de variáveis utilizadas apresentaram a variância acumulada (autovalores) de 94,65\% (Tabela 2), sendo, portanto, suficientes para representarem os dados originais com sucesso. Cada componente principal $\left(Z_{1}, Z_{2}\right.$ e $\left.Z_{3}\right)$ é uma combinação linear aditiva das 33 variáveis, tendo-se, por exemplo, para $\mathrm{Z}_{1}$ :

$$
\mathrm{Z}_{1}=0,0596 \mathrm{Ya}+0,2317 \mathrm{Tx}_{1}+\cdots-0,2021 \mathrm{ERS}_{4}
$$

Os resultados das análises de regressão dos coeficientes de Fourier, em função dos componentes principais, estão apresentados na Tabela 3. Verifica-se que as melhores regressões foram para $\mathrm{Y}_{0}, \mathrm{a}_{3} \mathrm{e}$ $\mathrm{a}_{4}$, cujos valores dos coeficientes de determinação $\left(\mathrm{R}^{2}\right)$ estão compreendidos entre 0,79 e 0,84 e os respectivos níveis críticos de probabilidades dos testes $\mathrm{F}$ da regressão com $\mathrm{P} \leq 0,1513$. Para os demais coeficientes, as regressões não se apresentaram estatisticamente satisfatórias, mostrando não existir boa resposta de regressão dos coeficientes de Fourier nos componentes principais das variáveis de entrada do modelo. Contudo, a eliminação dessas regressões não-significativas praticamente não alterou o resultado final do modelo. Assim, para que houvesse similaridade no conjunto de parâmetros entre o modelo prognóstico e o diagnóstico (modelo devido ao ajuste das séries de Fourier aos dados de produtividades), resolveu-se manter todas as regressões no modelo final. Dessa forma, o modelo originado com base na eq.(1) é representado pelo conjunto das seguintes equações:

$$
\begin{aligned}
& \hat{\mathrm{Y}}_{\text {est }}=\mathrm{Y}_{0}+\left[\left(\mathrm{a}_{1} \cos \left(1 \frac{2 \pi}{\mathrm{T}} \mathrm{t}_{\mathrm{i}}\right)+\left(\mathrm{b}_{1} \operatorname{sen}\left(1 \frac{2 \pi}{\mathrm{T}} \mathrm{t}_{\mathrm{i}}\right)\right]+\left[\left(\mathrm{a}_{2} \cos \left(2 \frac{2 \pi}{\mathrm{T}} \mathrm{t}_{\mathrm{i}}\right)+\left(\mathrm{b}_{2} \operatorname{sen}\left(2 \frac{2 \pi}{\mathrm{T}} \mathrm{t}_{\mathrm{i}}\right)\right]\right.\right.\right.\right. \\
& +\cdots+\left[\left(\mathrm{a}_{7} \cos \left(7 \frac{2 \pi}{\mathrm{T}} \mathrm{t}_{\mathrm{i}}\right)+\left(\mathrm{b}_{7} \operatorname{sen}\left(7 \frac{2 \pi}{\mathrm{T}} \mathrm{t}_{\mathrm{i}}\right)\right]\right.\right.
\end{aligned}
$$

em que, os coeficientes $Y_{0}, a_{n}$ e $b_{n}$ (" $n$ " variando de 1 a 7) são representados pelas equações seguintes, extraídas da Tabela 3:

$$
\begin{aligned}
& Y_{0}=1489,58+19,92 Z_{1}-75,73 Z_{2}-72,79 Z_{3} \\
& a_{1}=18,79-6,49 Z_{1}-2,62 Z_{2}+37,54 Z_{3} \\
& \dot{\cdot} \\
& \dot{b_{7}}=-40,38-14,12 Z_{1}+9,87 Z_{2}-4,66 Z_{3}
\end{aligned}
$$


ressaltando-se que $Z_{1}, Z_{2}$ e $Z_{3}$ são os componentes principais (funções lineares) das variáveis de entrada, conforme Tabela 2. O período (T) equivale a 15, devido ao fato de ser esse modelo gerado de séries compostas por 15 anos de dados de produtividades, enquanto $t_{i}$, ao valor de ordem do dado. Como esse modelo é de estimativa, $\mathrm{t}_{\mathrm{i}}$ será sempre igual a 1.

TABELA 2. Coeficientes de ponderação e variâncias dos componentes principais obtidos pela matriz de correlação das variáveis de entrada do modelo.

\begin{tabular}{|c|c|c|c|c|}
\hline \multirow{2}{*}{\multicolumn{2}{|c|}{ Variáveis }} & \multicolumn{3}{|c|}{ Coeficientes dos Componentes Principais } \\
\hline & & \multirow{2}{*}{$\begin{array}{c}\mathrm{Z}_{1} \\
0,0596\end{array}$} & \multirow{2}{*}{$\begin{array}{c}Z_{2} \\
-0,2267\end{array}$} & \multirow{2}{*}{$\begin{array}{c}Z_{3} \\
-0,2179\end{array}$} \\
\hline 1 & Ya & & & \\
\hline 2 & $\mathrm{Tx}_{1}$ & 0,2317 & $-0,0176$ & 0,1232 \\
\hline 3 & $\mathrm{Tx}_{2}$ & 0,2230 & $-0,1155$ & $-0,1032$ \\
\hline 4 & $\mathrm{Tx}_{3}$ & 0,0889 & $-0,2112$ & $-0,2627$ \\
\hline 5 & $\mathrm{Tx}_{4}$ & 0,2121 & $-0,1782$ & $-0,0294$ \\
\hline 6 & $\operatorname{Tn}_{1}$ & 0,0222 & $-0,2275$ & 0,2727 \\
\hline 7 & $\operatorname{Tn}_{2}$ & $-0,0015$ & $-0,3147$ & 0,1322 \\
\hline 8 & $\operatorname{Tn}_{3}$ & $-0,0419$ & $-0,3176$ & 0,0594 \\
\hline 9 & $\operatorname{Tn}_{4}$ & $-0,0590$ & $-0,2978$ & 0,1464 \\
\hline 10 & $\mathrm{~A}_{1}$ & 0,1606 & 0,2111 & $-0,1726$ \\
\hline 11 & $\mathrm{~A}_{2}$ & 0,2044 & 0,0986 & $-0,1798$ \\
\hline 12 & $\mathrm{~A}_{3}$ & 0,1276 & 0,0439 & $-0,3235$ \\
\hline 13 & $\mathrm{~A}_{4}$ & 0,1593 & 0,2137 & $-0,1604$ \\
\hline 14 & $\mathrm{Tm}_{1}$ & 0,2150 & $-0,0891$ & 0,1571 \\
\hline 15 & $\mathrm{Tm}_{2}$ & 0,2275 & $-0,1391$ & $-0,0276$ \\
\hline 16 & $\mathrm{Tm}_{3}$ & 0,1753 & $-0,2065$ & $-0,1373$ \\
\hline 17 & $\mathrm{Tm}_{4}$ & 0,1844 & $-0,2202$ & 0,0630 \\
\hline 18 & $\mathrm{P}_{1}$ & 0,2421 & 0,0509 & $-0,0891$ \\
\hline 19 & $\mathrm{P}_{2}$ & 0,0667 & 0,0405 & 0,3627 \\
\hline 20 & $\mathrm{P}_{3}$ & 0,1793 & 0,1463 & 0,1418 \\
\hline 21 & $\mathrm{P}_{4}$ & 0,2370 & 0,0944 & $-0,0144$ \\
\hline 22 & $\mathrm{ETR}_{1}$ & 0,2383 & 0,0882 & 0,0255 \\
\hline 23 & $\mathrm{ETR}_{2}$ & 0,2393 & $-0,0649$ & $-0,0009$ \\
\hline 24 & $\mathrm{ETR}_{3}$ & 0,1679 & $-0,1973$ & $-0,1695$ \\
\hline 25 & $\mathrm{ETR}_{4}$ & 0,2375 & $-0,0882$ & 0,0713 \\
\hline 26 & $\mathrm{DH}_{1}$ & $-0,0454$ & $-0,1562$ & 0,3259 \\
\hline 27 & $\mathrm{DH}_{2}$ & 0,1008 & $-0,2509$ & $-0,1842$ \\
\hline 28 & $\mathrm{DH}_{3}$ & $-0,1133$ & $-0,1839$ & $-0,1862$ \\
\hline 29 & $\mathrm{DH}_{4}$ & $-0,1546$ & $-0,2618$ & $-0,0476$ \\
\hline 30 & $\mathrm{ERS}_{1}$ & $-0,2021$ & $-0,0610$ & $-0,1788$ \\
\hline 31 & $\mathrm{ERS}_{2}$ & 0,2021 & 0,0610 & 0,1788 \\
\hline 32 & $\mathrm{ERS}_{3}$ & 0,2021 & 0,0610 & 0,1788 \\
\hline 33 & $\mathrm{ERS}_{4}$ & $-0,2021$ & $-0,0610$ & $-0,1788$ \\
\hline Variância (aut & & 15,62 & 8,85 & 6,76 \\
\hline Variância & & 47,33 & 26,83 & 20,48 \\
\hline Variação acum & & 47,33 & 74,16 & 94,65 \\
\hline
\end{tabular}


TABELA 3. Parâmetros, coeficientes de determinação e níveis de significância (teste F) das análises de regressões lineares múltiplas dos coeficientes das séries de Fourier, em função dos componentes principais.

\begin{tabular}{|c|c|c|c|c|c|c|}
\hline $\begin{array}{l}\text { Coeficiente } \\
\text { dos } \\
\text { Harmônicos }\end{array}$ & $\begin{array}{l}\text { Interseção } \\
\qquad\left(\beta_{0}\right)\end{array}$ & $\begin{array}{l}\mathrm{Z}_{1} \\
\left(\beta_{1}\right)\end{array}$ & $\begin{array}{l}\mathrm{Z}_{2} \\
\left(\beta_{2}\right)\end{array}$ & $\begin{array}{c}\mathrm{Z}_{3} \\
\left(\beta_{3}\right) \\
\end{array}$ & $\mathrm{R}^{2}$ & $\begin{array}{l}\text { Nível de Significância } \\
\text { (Teste F) }\end{array}$ \\
\hline $\mathrm{Y}_{0}$ & $1.489,58$ & 19,92 & $-75,73$ & $-72,79$ & 0,83 & 0,1115 \\
\hline$a_{1}$ & 18,79 & $-6,49$ & $-2,62$ & 37,54 & 0,69 & 0,2600 \\
\hline $\mathrm{b}_{1}$ & $-171,69$ & $-18,30$ & $-15,90$ & 72,39 & 0,38 & 0,6475 \\
\hline$a_{2}$ & $-30,35$ & $-1,25$ & $-22,42$ & 12,67 & 0,21 & 0,8480 \\
\hline$b_{2}$ & $-64,34$ & 2,77 & $-41,05$ & 0,14 & 0,59 & 0,3823 \\
\hline$a_{3}$ & $-144,28$ & 5,94 & 23,35 & 36,78 & 0,84 & 0,1024 \\
\hline $\mathrm{b}_{3}$ & 133,33 & 9,57 & $-19,17$ & 16,25 & 0,44 & 0,5776 \\
\hline $\mathrm{a}_{4}$ & $-45,03$ & 24,13 & $-24,27$ & 8,45 & 0,79 & 0,1513 \\
\hline $\mathrm{b}_{4}$ & 31,46 & 3,69 & 0,90 & 11,02 & 0,55 & 0,4355 \\
\hline$a_{5}$ & $-128,90$ & $-6,99$ & 0,51 & $-6,66$ & 0,17 & 0,8872 \\
\hline$b_{5}$ & 26,01 & 28,90 & $-19,98$ & 26,98 & 0,65 & 0,3137 \\
\hline$a_{6}$ & 42,90 & 20,84 & $-19,18$ & $-26,68$ & 0,46 & 0,5456 \\
\hline$b_{6}$ & 9,50 & 5,69 & 9,71 & 21,37 & 0,32 & 0,7195 \\
\hline$a_{7}$ & $-332,25$ & $-42,55$ & 30,84 & 24,68 & 0,67 & 0,2861 \\
\hline $\mathrm{b}_{7}$ & $-40,38$ & $-14,12$ & 9,87 & $-4,66$ & 0,11 & 0,9382 \\
\hline
\end{tabular}

Realizado o teste do modelo, observou-se que os erros relativos percentuais (ERP) de comparação entre valores estimados e observados variaram entre -39,54 e 85,58\% para os 15 anos de observações (Tabela 4). Os valores com sinal (-) indicam que as produtividades estimadas foram inferiores às observadas, enquanto os demais evidenciam que as estimativas foram superiores. Percebe-se, portanto, grande discrepância das estimativas.

$\mathrm{Na}$ Figura 1, ilustra-se o desempenho do modelo, indicando baixo valor do índice de WILLMOTT et al. (1985). Portanto, não apresenta boa concordância entre os valores estimados e observados, ou seja, com exatidão pouca expressiva, e, ainda, a regressão linear simples, com a reta passando pela origem, mostrou coeficiente de determinação muito baixo, o que indica a baixa precisão do modelo em estimar a produtividade do cafeeiro. Além desses baixos índices, verificou-se que, de modo geral, o modelo possui a tendência de subestimar os valores reais de produtividade.

Dentre os modelos desenvolvidos por PICINI (1998), apesar de menores discrepâncias, verificou-se, também, para aquele que apresentou melhor resultado, variações ao comparar as produtividades estimadas versus observadas, cujos valores de " $\mathrm{r}^{2}$ " e do índice de concordância "d" da regressão foram, respectivamente, 0,85 e 0,50 para a região de Campinas, 0,46 e 0,78 para a região de Gália e 0,93 e 0,98 para a região de Mococa, no Estado de São Paulo.

Embora o modelo proposto tenha apresentado grandes discrepâncias nas estimativas da produtividade de café, foi verificado certo acompanhamento das produtividades estimadas em relação às observadas. Assim, desde que se encontrem alternativas para diminuir as discrepâncias exibidas, a metodologia pode viabilizar a modelagem de previsão de safras da cultura do café, contribuindo, portanto, com subsídios para os estudos de modelos para essa cultura. É evidente que, além dos elementos climáticos, outros fatores podem afetar a produção cafeeira. $\mathrm{O}$ comportamento do mercado, condições adversas do clima, o surgimento de novas tecnologias de manejo e variedades para a cultura do café afetam, consideravelmente, a produção cafeeira, tornando-a bastante instável ao longo dos anos. Esses fatores indicam o nível de complexidade para elaborar modelos de previsão de safras 
cafeeiras. Deve-se salientar que o modelo proposto não leva em consideração essas situações adversas e, logicamente, não é adequado para fazer previsão de produtividade nessas condições.

TABELA 4. Resultados das produtividades $\left(\mathrm{kg} \mathrm{ha}^{-1}\right)$ observadas (Yobs) e estimadas (Yest), e erros relativos percentuais (ERP) das estimativas para o município de Três Pontas - MG.

\begin{tabular}{rrrrr}
\hline Ano & Ano Agrícola & Yobs & Yest & \multicolumn{1}{c}{ ERP } \\
\hline 1985 & $1984 / 1985$ & 1.387 & - & - \\
1986 & $1985 / 1986$ & 651 & 1.208 & 85,58 \\
1987 & $1986 / 1987$ & 2.054 & 1.373 & $-33,16$ \\
1988 & $1987 / 1988$ & 1.168 & 1.810 & 55,06 \\
1989 & $1988 / 1989$ & 1.378 & 1.226 & $-11,01$ \\
1990 & $1989 / 1990$ & 986 & 1.051 & 6,55 \\
1991 & $1990 / 1991$ & 1.230 & 829 & $-32,64$ \\
1992 & $1991 / 1992$ & 1.000 & 1.331 & 33,06 \\
1993 & $1992 / 1993$ & 1.654 & 1.013 & $-38,72$ \\
1994 & $1993 / 1994$ & 1.254 & 1.339 & 6,79 \\
1995 & $1994 / 1995$ & 955 & 1.004 & 5,18 \\
1996 & $1995 / 1996$ & 1.632 & 1.315 & $-19,42$ \\
1997 & $1996 / 1997$ & 1.632 & 1.619 & $-0,81$ \\
1998 & $1997 / 1998$ & 1.632 & 1.684 & 3,21 \\
1999 & $1998 / 1999$ & 1.632 & 1.071 & $-34,41$ \\
2000 & $1999 / 2000$ & 1.632 & 987 & $-39,54$ \\
\hline
\end{tabular}

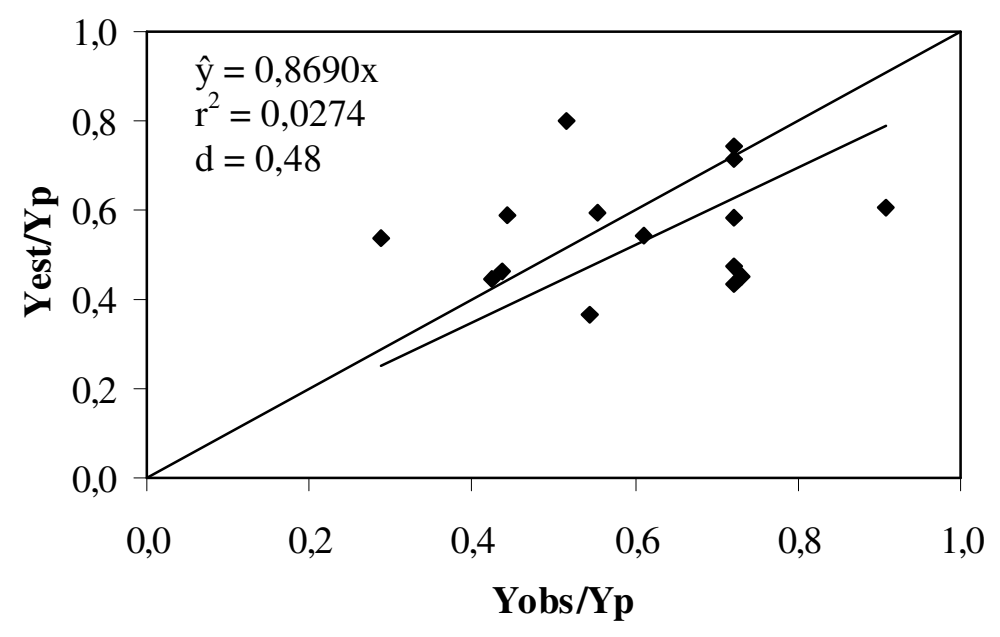

FIGURA 1. Produtividades relativas estimadas (Yest/Yp) e observadas (Yobs/Yp) e respectivo desempenho estatístico do modelo prognóstico de produtividade da cultura do café testado para o município de Três Pontas - MG.

\section{CONCLUSÃO}

O modelo não se mostrou satisfatório na previsão de produtividades para a cultura do café.

\section{REFERÊNCIAS}

ALVES, A.R.; VIANELLO, R.L.; SEDIYAMA, G.C.; COELHO, D.T. Determinação analítica dos instantes do "nascer" e do "pôr-do-sol" para superfícies inclinadas quaisquer. Ciência e Cultura, São Paulo, v.35, n.2, p.194-8, 1983. 
ASPIAZÚ, C.; RIBEIRO, G.A.; VIANELLO, R.L.; RIBEIRO, J.C.; VALENTE, O.F.; PAULA NETO, F. de. Análise dos componentes principais aplicada na classificação climática do Estado de Minas Gerais. Teste metodológico. Revista Árvore, Viçosa, v.14, n.1, p.1-15, 1990.

ASSIS, F.N. de; ARRUDA, H.V. de; PEREIRA, A.R. Aplicações de estatística à climatologia: teoria e prática. Pelotas: Ed. Universitária/UFPel, 1996. 161 p.

CAMARGO, A.P. Balanço hídrico, florescimento e necessidade de água para o cafeeiro. In: SIMPÓSIO SOBRE O MANEJO DE ÁGUA NA AGRICULTURA, 1987, Campinas. Anais... Campinas: Fundação Cargill, 1987. p.53-90.

CRUZ, C.D.; REGAZZI, A.J. Modelos biométricos aplicados ao melhoramento genético. 2.ed. Revisada. Viçosa - MG: Editora UFV, 1997. 390 p.

HAAN, C.T. Statistical methods in hidrology. $5^{\text {th }}$ ed. Ames: The Iowa State University Press, 1977. $378 \mathrm{p}$.

HOOGENBOOM, G. Contribution of agrometeorology to the simulation of crop production and its applications. Agricultural and Forest Meteorology, Amsterdam, v.103, p.137-57, 2000.

KANEMASU, E.T. Yield and water-use relationships: some problems of relating grain yield to transpiration. In: TAYLOR, H.M.; JORDAN, W.R.; SINCLAIR, T.R. (Ed.). Limitations to efficient water user in crop production. Madison: American Society of Agronomy, 1983. cap. 9B, p.413-17.

MATIELLO, J.B. O café: do cultivo ao consumo. São Paulo: Editora Globo, 1991.319 p. (Coleção do Agricultor - Grãos).

PEREIRA, A.R.; VILLA NOVA, N.A.; SEDIYAMA. G.C. Evapo(transpi)ração. Piracicaba: FEALQ, 1997. 183 p.

PICINI, A.G. Desenvolvimento e teste de modelos agrometeorológicos para a estimativa de produtividade do cafeeiro (Coffea arabica L.) a partir do monitoramento da disponibilidade hídrica do solo. 1998. 132 f. Dissertação (Mestrado em Agronomia) - Escola Superior de Agricultura "Luiz de Queiroz”, Universidade de São Paulo, Piracicaba, 1998.

RENA, A.B.; MAESTRI, M. Fisiologia do cafeeiro. Informe Agropecuário, Belo Horizonte, v.11, n.126, p.26-40, 1985.

STEWART, J.I.; HAGAN, R.M.; PRUITT, W. Production functions and predicted irrigation programmes for principal crops as required for water resources planning and increased water use efficiency: final report. Washington: U.S. Department of Interior, 1976.

WEILL, M.A.M. Avaliação de fatores edafoclimáticos e do manejo na produção de cafeeiros (Coffea arabica L.) na região de Marília e Garça, SP. 1990. 182 f. Dissertação (Mestrado) - Escola Superior de Agricultura "Luiz de Queiroz”, Universidade de São Paulo, Piracicaba, 1990.

WILLMOTT, C.J.; ACKLESON, S.G.; DAVIS, R.E.; FEDDEMA, J.J.; KLINK, K.M.; LEGATES, D.R.; O'DONNELL, J.; ROWE, C.M. Statistics for the evaluation and comparison of models. Journal of Geophysical Research, Washington, v.90, n.C5, p.8995-9005, 1985. 